\title{
Anomalous resistivity in beam-return currents and hard- $X$ ray spectra of solar flares
}

\author{
L. $\mathrm{Xu}^{1,2}, \mathrm{~L} . \mathrm{Chen}^{1}$, and D. J. Wu ${ }^{1}$ \\ 1 Purple Mountain Observatory, CAS, Nanjing 210008, PR China \\ e-mail: djwu@pmo.ac.cn \\ ${ }^{2}$ University of Chinese Academy of Sciences, Beijing 100049, PR China
}

Received 18 August 2012 / Accepted 3 December 2012

\section{ABSTRACT}

\begin{abstract}
Context. Observations of hard-X ray (HXR) spectra from solar flares show that there is noncollisional energy loss when energetic beam electrons are transported along the flare loop from their acceleration site above the looptop in the corona to the loop footpoints in the chromosphere.

Aims. This paper investigates anomalous (i.e., noncollisional) resistivity due to the effective collision by the wave-particle interaction in the beam-return current system of a flare and its effect on the HXR spectral evolution between the looptop and footpoint sources. Methods. To attribute the noncollisional energy loss to an induced electric field by the beam current, the induced electric field is estimated by the spectral evolution between the looptop and footpoint sources, which is deduced from the standard thin-thick target model. To include collisional and anomalous resistivity caused by the ion-acoustic wave turbulence excited by the return current, the necessary excited level and the excited condition are discussed for the steady-state case in which the return current density driven by the induced electric field in terms of Ohm's law is required to be equal to the beam current density.

Results. The results show that including the anomalous resistivity can reasonably remove the discrepancy between observations and predictions. Meanwhile, the necessary excited level for the ion-acoustic turbulence is tens times of the thermal noise of electrostatic fluctuations in the background plasma, which is an ordinary and low excited level that is easily satisfied.

Conclusions. This indicates that the microscopic kinetics of plasma particles possibly play an important and critical role in understanding the dynamics of beam-return current systems in the solar atmosphere and in the physics of solar flares.
\end{abstract}

Key words. Sun: flares - Sun: X-rays, gamma rays - plasmas - acceleration of particles

\section{Introduction}

It is commonly believed that the hard X-ray (HXR) emission of solar flares is attributed to bremsstrahlung emission produced by energetic electrons accelerated somewhere above the flare loop. The HXR sources in observation can be featured frequently by a looptop source in the corona (Frost \& Dennis 1971; Hudson 1978) and two or more footpoint sources in the chromosphere (Hoyng et al. 1981). It is frequently suggested that both looptop and footpoint sources are caused by a single energetic electron beam that travels along the flare loop from the looptop to the footpoint. The standard model for the HXR emission involves the thin-target emission at the looptop and the thick-target emission from the footpoints. In this standard model a power-law spectrum of energetic beam electrons in the looptop

$F_{\text {tp }}(\varepsilon) \propto \varepsilon^{-\delta_{\text {tp }}}$

with a spectral index $\delta_{\text {tp }}$ will emit HXR with a power-law spectrum

$I_{\mathrm{tp}}(\epsilon) \propto \epsilon^{-\gamma_{\mathrm{tp}}}$,

but with a spectral index $\gamma_{\mathrm{tp}}=\delta_{\mathrm{tp}}+1$, via thin-target emission (Arnoldy et al. 1968; Holt \& Cline 1968; Takakura 1969; Lin $\&$ Hudson 1976). If without energy loss, the same beam electrons will produce a power-law HXR spectrum with an index of $\gamma_{\mathrm{ft}}=\delta_{\mathrm{ft}}-1=\delta_{\mathrm{tp}}-1$ via the thick-target emission (Brown 1971; Syrovatskii \& Shmeleva 1972) when they travel to the chromosphere at the footpoint. The standard flare model thus predicts the difference between the observed spectral indices of the looptop, and footpoint HXR sources as follows:

$\Delta \gamma=\gamma_{\mathrm{tp}}-\gamma_{\mathrm{ft}}=2$.

However, the majority of observations of solar flare events with the predicted spectral index difference do not demonstrate this behavior (Masuda et al. 1994, 1995, 2000; Petrosian et al. 2002). Apparently, it is expected that high-resolution observations of the HXR emissions of beam electrons at the looptop and at the footpoint sources and the comparison between their spectra will provide well-constrained information about the acceleration and traveling processes of the beam electrons. The Ramaty High Energy Solar Spectroscopic Imager (RHESSI, Lin et al. 2002) provides the possibility of making high-resolution imaging spectroscopy at different locations on the sun. Therefore, RHESSI observations provide routine availability to study each source separately in events with several contemporaneous HXR-sources at spatially resolved spectroscopy with high-energy resolution, and it has triggered a number of interesting findings (Emslie et al. 2003; Battaglia \& Benz 2006, 2007, 2008; Saint-Hilaire et al. 2008; Su et al. 2009).

To study the spectral evolution of HXR emissions between the looptop and footpoint sources, Battaglia \& Benz (2006) have analyzed five flares that possess HXR spectra of simultaneous looptop and footpoint sources and find a range of $0<\Delta \gamma<6$ for the index difference between their looptop and footpoint HXR spectra. In particular, they find that in two of those events, 
the differences at specific times, as well as the time-averaged difference, were significantly more than two, ruling out a simple thin-thick target interpretation. A possible explanation for the discrepancy is that there was an anomalously high density concentrated at the looptop source in the corona so that this emission is a combination of thin-thick target, which would result in a flatter footpoint spectral index at low energies, namely $\Delta \gamma>2$ (Battaglia \& Benz 2007), as previously suggested by Wheatland \& Melrose (1995) for the coronal HXR sources observed by Yohkoh/HXT (Masuda et al. 1994; Feldman et al. 1994). However this model predicts a break in the coronal HXR spectrum between the thin and thick target range, which has not been observed with RHESSI data despite its high energy resolution.

An alterative suggestion by Battaglia \& Benz (2008) was to include noncollisional energy losses of the beam electrons when traveling downwards to the chromosphere via a decelerating electric potential, whose electric field was attributed to the return current induced by the electron beam in terms of Ohm's law for a finite conductivity case. They selected two of the five events analyzed by Battaglia \& Benz (2006), where the index differences were significantly greater than two. Their results show that this noncollisional energy loss by means of the induced electric field did flatten the footpoint spectrum and led to the necessary larger difference between spectral indices, provided that the induced electric field is maintained at proper strength. In a steady-state case, the return current can be estimated by Ohm's law if assuming the Spitzer conductivity. Unfortunately, for the two events analyzed by Battaglia \& Benz (2008), the resulting return current is an order of magnitude higher than the beam current. This obviously contradicts the assumption of a steady state, so it is unphysical, too.

To assume a nonsteady state, Hannah \& Kontar (2011) investigated the spectral difference between solar flare HXR coronal and footpoint sources due to wave-particle interactions. With a transiently injected beam of accelerated electrons, their numerical results show that the wave-particle interactions dominate in the corona, whereas the collisional losses dominate in the chromosphere. In particular, the addition of wave-particle interactions to collisional transport for a transient, initially injected electron beam flattens the spectrum of the footpoint source. This requires a transiently injected electron beam to produce significant wave growth, but entirely neglects the role of return currents. However, this transient process is valid only for the initial stage of the dynamics of an injected electron beam.

In fact, the characteristic time for the wave growth is much less than the traveling time of the beam electrons from the looptop to footpoint sources. As a result, the propagation of the electron beam rapidly relaxes to a steady state. When a beam of accelerated electrons propagates along the loop from the looptop in the corona to the footpoint at the chromosphere, if it is not balanced by an equal beam of ions, a return current is inevitably established to prohibit the charge from building up and a beam-associated magnetic field from being induced. The formation and evolution of the return current and the electrodynamics of the beam-return current system have been studied by many authors (e.g. Knight \& Sturrock 1977; Spicer \& Sudan 1984; Larosa \& Emslie 1989; van den Oord 1990). In the return current, background thermal or suprathermal electrons move towards the looptop source with a relatively low velocity. These return electrons collide with background ions and cause resistivity. Ohms law implies the presence of an induced electric field in the downward direction, which provides a decelerating electric potential for the beam electrons propagating downwards.
In this paper, to include effects of an anomalous resistivity due to the wave-particle interaction we analyze Ohm's law in a steady-state beam-return current system, in which the anomalous resistivity can be much larger than the collisional resistivity in the observed parameter regimes. This indicates much less anomalous conductivity than the Spitzer conductivity, hence results in the return current that is much less than that estimated by Omh's law when assuming the Spitzer conductivity. In particular, for the two events presented by Battaglia \& Benz (2008), this removes the contradiction that the estimated return current is an order of magnitude higher than the beam current.

\section{Effective collisional frequency and anomalous resistivity}

The injection of a beam of energetic electrons into a plasma drives a charge- and current-neutralizing return current in the plasma (Hoyng et al. 1976; van den Oord 1990). The return current is driven by an electric field induced by the beam current of the energetic electrons in the plasma (van den Oord 1990). Then, in addition to driving the return current, this induced electric field also extracts energy from the beam electrons and collisionally heats the plasma through Joule heating. In the steady state this return current is governed by Ohm's law, that is,

$j_{\mathrm{r}}=\sigma E_{\mathrm{i}}$,

where $E_{\mathrm{i}}$ is the induced electric field, $\sigma$ the conductivity, and $j_{\mathrm{r}}$ the return current density, which is required to balance the beam current density $j_{\mathrm{b}}$ and to maintain the current neutralization for the steady-state case in terms of $j_{\mathrm{r}}=j_{\mathrm{b}}$ but with a reverse direction.

When to neglect instabilities of beam-plasma interactions and possibility of turbulent waves, the return current, in general, is considered to be formed by the major electrons of the background plasma, which have the same drifting velocity $v_{\mathrm{d}}$ (Knight \& Sturrock 1977; Hoyng et al. 1978; Spicer \& Sudan 1984; van den Oord 1990; Bret 2009; Karlický 2009). The conductivity $\sigma$ is mainly contributed by the Spitzer conductivity via the classical Coulomb collision, that is,

$\sigma=\sigma_{\mathrm{c}}=\frac{n_{0} e^{2}}{m_{\mathrm{e}} v_{\mathrm{c}}}=\frac{\epsilon_{0} \omega_{\mathrm{pe}}^{2}}{v_{\mathrm{c}}} \simeq 500 T_{\mathrm{e}}^{3 / 2}$

with the electron collision frequency (Huba 2004)

$v_{\mathrm{c}} \simeq 5.8 \times 10^{-5} \frac{n_{0}}{T_{\mathrm{e}}^{3 / 2}}$,

where the background electron temperature $T_{\mathrm{e}}$ and density $n_{0}$ are in units of $\mathrm{eV}$ and $\mathrm{cm}^{-3}$, respectively, and the Coulomb logarithm $\ln \Lambda \simeq 20$ has been used for the sake of simplification.

However, due to having a higher beam speed $\left(v_{\mathrm{b}}\right)$ than the thermal speed of the background electrons $\left(v_{T_{\mathrm{e}}}\right)$, the beam electrons in a solar flare often are instable to beam-plasma interactions and lead to strong Langmuir turbulence (Vlahos \& Rowland 1984; Zharkova \& Siversky 2011). The Langmuir turbulence effectively heats a minor part of the background electrons to suprathermal electrons to form suprathermal tails of the background thermal electrons, which can escape from the strong turbulence (Papadopoulos \& Rowland 1978). As pointed out by Rowland \& Vlahos (1985), this can considerably modify the way the return current is carried by the bulk of the background electrons. Instead of this, the return current is carried by 
the runaway electrons that are not trapped in the strong turbulence. Their results show that the majority of the plasma electrons are trapped and the return current is carried by a small number of suprathermal electrons for a broad range of beam densities, $10^{-4}<n_{\mathrm{b}} / n_{0}<10^{-1}$, with the typical beam electron velocity $v_{\mathrm{b}}=10 v_{T_{\mathrm{e}}}$, where $n_{\mathrm{b}}\left(n_{0}\right)$ is the beam (plasma) density and $v_{T_{\mathrm{e}}}$ is the thermal speed of the background electrons.

Thus, the collisional losses are reduced and the drift velocity $v_{\mathrm{d}}=j_{\mathrm{r}} /\left(e n_{\mathrm{r}}\right)$ of return current electrons is increased, where $n_{\mathrm{r}}$ is the density of suprathermal electrons that carry the return current. In particular, when the drifting velocity of return current electrons exceeds a critical value, the return current becomes unstable and excites ion-acoustic wave turbulence (Spicer 1977; Cromwell et al. 1988). This leads to the presence of anomalous collisions and the corresponding anomalous resistivity (i.e., the so-called Sagdeev resistivity) via the wave-particle interaction between the return current electrons and the ion-acoustic waves, with an effective collision frequency (Sagdeev 1967; Sagdeev \& Galeev 1969; Hasegawa 1975; Papadoplous 1977; Galeev \& Sagdeev 1984; Labelle \& Treumann 1988; Benz 2002)

$v_{\mathrm{eff}} \simeq \frac{W_{\mathrm{ia}}}{n_{0} T_{\mathrm{e}}} \omega_{\mathrm{pe}}$

where $W_{\mathrm{ia}}$ is the wave energy density of the ion-acoustic turbulence.

The current-driven ion-acoustic instability and the effects caused by the corresponding anomalous resistivity have been extensively discussed by many authors (Sagdeev 1967; Sagdeev \& Galeev 1969; Hasegawa 1975; Spicer 1977; Papadoplous 1977; Galeev \& Sagdeev 1984; Cromwell et al. 1988; Labelle \& Treumann 1988; Benz 2002). The ion-acoustic wave energy density at saturation depends on the plasma electron to ion temperature ratio, $T_{\mathrm{e}} / T_{\mathrm{i}}$, as well as the ratio of the drift velocity of the carrying electrons to the thermal velocity of the plasma electrons, $v_{\mathrm{d}} / v_{T_{\mathrm{e}}}$, (Sagdeev 1967; Papadolous 1977; Benz 2002). Using the weak-turbulence theory, Sagdeev calculated the effective collisional frequency at the saturation, and the result reads as

$v_{\mathrm{eff}}^{\mathrm{S}} \simeq \frac{\omega_{\mathrm{pe}}}{32 \pi} \frac{T_{\mathrm{e}}}{T_{\mathrm{i}}} \frac{v_{\mathrm{d}}}{v_{T_{\mathrm{e}}}}$.

In combination with Eq. (7), the saturation level of the ionacoustic turbulence is given by

$$
\frac{W_{\mathrm{ia}}^{\mathrm{S}}}{n_{0} T_{\mathrm{e}}} \simeq \frac{1}{32 \pi} \frac{T_{\mathrm{e}}}{T_{\mathrm{i}}} \frac{v_{\mathrm{d}}}{v_{T_{\mathrm{e}}}} .
$$

On the other hand, as noticed by Hasegawa (1975), the classical Coulomb collision frequency $v_{\mathrm{c}}$ can be approximately given by

$$
v_{\mathrm{c}} \simeq \frac{\omega_{\mathrm{pe}}}{N_{\mathrm{D}}}=\frac{W_{\mathrm{ia}}^{\mathrm{T}}}{n_{0} T_{\mathrm{e}}} \omega_{\mathrm{pe}},
$$

where $N_{\mathrm{D}}=n_{0} \lambda_{\mathrm{D}}^{3}$ is the particle number within a Debye volume with the Debye length $\lambda_{\mathrm{D}}, W_{\mathrm{ia}}^{\mathrm{T}}=n_{0} T_{\mathrm{e}} / N_{\mathrm{D}}$ the energy density of the electrostatic fluctuation at the thermal noise level, that is, the energy density of ion-acoustic noise in a thermal equilibrium plasma (Hasegawa 1975; Benz 2002). To combine Eqs. (7) and (10), one can rewrite the effective collision frequency $v_{\mathrm{eff}}$ as

$$
v_{\mathrm{eff}} \simeq \frac{W_{\mathrm{ia}}}{W_{\mathrm{ia}}^{\mathrm{T}}} v_{\mathrm{c}}=\alpha v_{\mathrm{c}}
$$

implying that the effective to collision frequency ratio is directly proportional to the energy density ratio of the turbulence to the thermal noise, $\alpha \equiv W_{\mathrm{ia}} / W_{\mathrm{ia}}^{\mathrm{T}}$, which represents the excited level of the ion-acoustic turbulence. Because ordinarily $N_{\mathrm{D}}$ is a very large number with orders of $10^{4}-10^{8}$, the electrostatic fluctuation at the thermal noise level has a very low energy density $W_{\mathrm{ia}}^{\mathrm{T}} / n_{0} T_{\mathrm{e}} \sim 10^{-4}-10^{-8}$. While the saturation level of the weak ion-acoustic turbulence can be estimated as $\alpha_{\mathrm{S}}=W_{\mathrm{ia}}^{\mathrm{S}} / W_{\mathrm{ia}}^{\mathrm{T}} \sim 10^{1}-10^{5} \gg 1$ from Eq. (9), where the relations $T_{\mathrm{e}} \sim T_{\mathrm{i}}$ and $v_{\mathrm{d}} / v_{T_{\mathrm{e}}} \sim 0.1$ have been used. The ion-acoustic turbulence, therefore, is very easy excited so that $\alpha_{\mathrm{S}}>\alpha>\alpha_{\mathrm{T}} \equiv$ $W_{\text {ia }}^{\mathrm{T}} / W_{\text {ia }}^{\mathrm{T}}=1$, hence $v_{\text {eff }}>v_{\mathrm{c}}$.

To include the effective collision frequency, the conductivity in Ohm's law (4) $\sigma$ is replaced by

$\sigma=\frac{\epsilon_{0} \omega_{\mathrm{pe}}^{2}}{v_{\mathrm{c}}+v_{\mathrm{eff}}}=\frac{\sigma_{\mathrm{c}}}{1+\alpha}$.

This leads to the actual return current density in Ohm's law

$j_{\mathrm{r}}=\sigma E_{\mathrm{i}}=\frac{j_{\mathrm{cr}}}{1+\alpha}$

which is lower than the value estimated by the Spitzer conductivity, $j_{\mathrm{cr}}=\sigma_{\mathrm{c}} E_{\mathrm{i}}$, for the same induced electric field $E_{\mathrm{i}}$.

\section{Induced electric field and return current}

As proposed by Battaglia \& Benz (2008), the induced electric field $E_{\mathrm{i}}$ can be estimated by the spectral evolution of the beam electrons from the looptop source to the footpoint source because the induced electric field causes the beam electrons to lose energy when they travel from the looptop source to the footpoint source by means of its electric potential drops.

On the basis of RHESSI observations, Battaglia \& Benz (2008) analyzed the two solar flares on Oct. 24, 2003 and Jul. 13, 2005, whose differences in nonthermal HXR spectral index between the looptop and footpoint sources are significantly greater than two (Battaglia \& Benz 2007). They suggest that when the beam electrons travel from the looptop source in the corona to the footpoint source in the chromosphere, they can lose energy by means of the electric potential difference $\left(\Delta \Phi=L E_{\mathrm{i}}\right)$ of the induced electric field $E_{\mathrm{i}}$ between the looptop and footpoint sources. The energy loss of the beam electrons in the footpoint source can be estimated by

$\varepsilon_{\mathrm{ls}}=e \Delta \Phi=e L E_{\mathrm{i}}$,

where $L$ is half of the loop length (i.e., the distance between the looptop and footpoint sources along the loop).

Assuming the energy loss of the beam electrons by the induced electric field, $\varepsilon_{\mathrm{ls}}$, to be independent of their initial energy, $\varepsilon^{\prime}$, at the looptop source, the energy distribution of the beam electrons evolves from the initial form

$F_{\mathrm{tp}}\left(\varepsilon^{\prime}\right)=A \varepsilon^{\prime-\delta_{\mathrm{tp}}}$

at the looptop source to the final form experienced energy loss

$F_{\mathrm{ft}}(\varepsilon)=A\left(\varepsilon+\varepsilon_{\mathrm{ls}}\right)^{-\delta_{\mathrm{tp}}}$

at the footpoint source, which is flatter, although not strictly a power-law function anymore, where $A$ is a normalized constant and $\varepsilon=\varepsilon^{\prime}-\varepsilon_{\mathrm{ls}}$ is the energy of the beam electrons when they arrive at the footpoint source. As a consequence, a smaller spectral index is obtained for the beam electrons at the footpoints, that is, 
$\delta_{\mathrm{ft}}<\delta_{\mathrm{tp}}$, implying that the index difference is deduced from the thin-thick target model

$\Delta \gamma=\gamma_{\mathrm{tp}}-\gamma_{\mathrm{ft}}=2+\delta_{\mathrm{tp}}-\delta_{\mathrm{ft}}>2$.

It is obvious that the fitted spectral index of the beam electrons at the footpoint source $\delta_{\mathrm{ft}}$ (hence the corresponding thick-target photo spectral index $\left.\gamma_{\mathrm{ft}}=\delta_{\mathrm{ft}}-1\right)$ depends on the initial spectral index of the beam electrons at the looptop source $\delta_{\mathrm{tp}}$ (or the corresponding thin-target photo spectral index $\left.\gamma_{\mathrm{tp}}=\delta_{\mathrm{tp}}+1\right)$, as well as the experienced energy loss $\varepsilon_{\mathrm{ls}}$. As a simplification, the energy spectrum of the beam electrons at the footpoints can be fitted with a single power-law spectrum. At the looptop source, however, the case is more complex, in particular in the low energy band lower than $25 \mathrm{keV}$ because of the influence from the thermal component. Using the two different fitting methods for the low energy band as an estimation of the uncertainty (the one is to fit the low energy spectrum with a thermal component and the other is to fit the low energy spectrum with the thermal full sun as an approximation to the coronal thermal component), Battaglia \& Benz (2008) obtained an average index difference of $\Delta \gamma=3.55 \pm 0.07$ for the event on Oct. 24, 2003 and $\Delta \gamma=2.45 \pm 0.35$ for the event on July 13, 2005. To fit these observed looptop and footpoint spectra, they derived a range of the necessary energy loss, by using the different fitting methods, $\varepsilon_{\mathrm{ls}}=(58.0-59.4) \mathrm{keV}$ for the event on Oct. 24, 2003 and $\varepsilon_{\mathrm{ls}}=(8.7-26) \mathrm{keV}$ for the event on July 13, 2005.

The induced electric field $E_{\mathrm{i}}$ can be obtained from the necessary energy losses and the estimated distance between the looptop and footpoint sources (i.e., half of the loop length $L$ ), and the result reads as

$E_{\mathrm{i}}=\frac{\varepsilon_{\mathrm{ls}}}{e L}=(1.81-1.86) \times 10^{-3} \mathrm{~V} / \mathrm{m}$

for the event on Oct. 24, 2003 with $L \sim 3.2 \times 10^{7} \mathrm{~m}$ and

$E_{\mathrm{i}}=\frac{\varepsilon_{\mathrm{ls}}}{e L}=(2.02-6.05) \times 10^{-4} \mathrm{~V} / \mathrm{m}$

for the event on July 13, 2005 with $L \sim 4.3 \times 10^{7} \mathrm{~m}$.

Following Battaglia \& Benz (2008), to assume an average loop plasma temperature $T \sim 15 \mathrm{MK} \simeq 1.293 \mathrm{keV}$ which is between the looptop temperature and the footpoint temperature, one has the return current density $j_{\text {cr }}$ that is estimated by the Spitzer conductivity of Eq. (5), to read as

$j_{\text {cr }}=\sigma_{\mathrm{c}} E_{\mathrm{i}}=(4.12-4.24) \times 10^{4} \mathrm{~A} / \mathrm{m}^{2}$

for the event on Oct. 24, 2003, and

$j_{\mathrm{cr}}=\sigma_{\mathrm{c}} E_{\mathrm{i}}=(0.46-1.38) \times 10^{4} \mathrm{~A} / \mathrm{m}^{2}$

for the event on July 13, 2005.

On the other hand, the range of the beam current density can be derived from the RHESSI data (Battaglia \& Benz 2008). On the basis of Eqs. (10) and (11) of Battaglia \& Benz (2008), the values for the beam current density we present here differ slightly from the ones cited by Battaglia \& Benz (2008) because they used an effective column density for calculating the total electron flux, instead of the column density calculated directly based on Table 2 of Battaglia \& Benz (2008). By combining Eqs. (10) and (11) of Battaglia \& Benz (2008), we can obtain the expression for the beam current density $j_{\mathrm{b}}$ as follows:

$j_{\mathrm{b}}=\frac{e F_{\mathrm{tot}}}{A_{\mathrm{ft}}}=\frac{e A}{(\delta-1) A_{\mathrm{ft}} \varepsilon_{\mathrm{cut}}^{\delta-1}}$, where $A$ and $\delta$ are the normalized constant and the spectral index, respectively, for the electron spectral flux of the beam electrons $F(\varepsilon)=A \varepsilon^{-\delta}, A_{\mathrm{ft}}$ is the footpoint area, $\varepsilon_{\text {cut }}$ the electron cut energy, and $F_{\text {tot }}=\int_{\varepsilon_{\text {cut }}}^{\infty} F(\varepsilon) \mathrm{d} \varepsilon=A \varepsilon_{\text {cut }}^{1-\delta} /(\delta-1)$ the total electron flux per second. By use of the parameters presented in Table 2 of Battaglia \& Benz (2008), we obtained a range for the estimated beam current density corresponding to that of the observed electron spectral flux $F(\varepsilon)$ as follows:

$j_{\mathrm{b}}=(1.15-2.72) \times 10^{3} \mathrm{~A} / \mathrm{m}^{2}$

for the event on Oct. 24, 2003 and

$j_{\mathrm{b}}=(1.25-1.31) \times 10^{3} \mathrm{~A} / \mathrm{m}^{2}$

for the event on July 13, 2005.

To compare with above $j_{\mathrm{cr}}$, one has

$\frac{j_{\text {cr }}}{j_{\mathrm{b}}}=15.6-35.8 \Rightarrow \frac{\left\langle j_{\text {cr }}\right\rangle}{\left\langle j_{\mathrm{b}}\right\rangle} \sim 21.6$

for the event on Oct. 24, 2003 and

$\frac{j_{\mathrm{cr}}}{j_{\mathrm{b}}}=3.68-10.5 \Rightarrow \frac{\left\langle j_{\mathrm{cr}}\right\rangle}{\left\langle j_{\mathrm{b}}\right\rangle} \sim 7.2$

for the event on July 13, 2005, where \langle\rangle denotes the average estimated by the two different methods (Battaglia \& Benz 2008). As pointed out by Battaglia \& Benz (2008), with the Spitzer conductivity $\sigma_{\mathrm{c}}$ caused by only the classical Coulomb collision $v_{\mathrm{c}}$, the return current $j_{\text {cr }}$ is an order of magnitude higher than the beam current $j_{\mathrm{b}}$, which contradicts the assumptions of a steady state and is also unphysical, because some important processes possibly have been missed, for instance, beam-plasma (or current-plasma) instabilities and wave-particle interactions.

To include the effective collision frequency due to the waveparticle interaction by the ion-acoustic wave turbulence, the actual return current $j_{\mathrm{r}}$ is given by Eq. (13), which can be much less than $j_{\text {cr }}$ when $\alpha \gg 1$, that is, the ion-acoustic turbulence is very excited in the plasma. For the steady-sate case in which the relation $j_{\mathrm{r}}=j_{\mathrm{b}}$ is required by the current neutralizing condition, one has the necessary excited level of the ion-acoustic turbulence from Eq. (13)

$\alpha \equiv \frac{W_{\mathrm{ia}}}{W_{\mathrm{ia}}^{\mathrm{T}}}=\frac{\left\langle j_{\mathrm{cr}}\right\rangle}{\left\langle j_{\mathrm{b}}\right\rangle}-1$

to lead to $\alpha \sim 20$ for the event on Oct. 24, 2003 and $\alpha \sim 7$ for the event on July 13, 2005, which both are low saturation levels, and can easy be satisfied (Spicer 1977; Cromwell et al. 1988).

For the estimated loop plasmas with $T_{\mathrm{e}}=15 \mathrm{MK}$ in both on Oct. 24, 2003 and Jul. 13, 2005 flare events, one has approximately the electron thermal velocity $v_{T_{\mathrm{e}}} \simeq 1.5 \times 10^{7} \mathrm{~m} / \mathrm{s}$ and the ion-acoustic velocity $c_{\mathrm{s}} \simeq 3.5 \times 10^{5} \mathrm{~m} / \mathrm{s}$. The average velocity of the beam electrons depends on the spectral index $\delta$, as well as on the cutoff energy $\varepsilon_{\text {cut }}$, and is estimated as $v_{\mathrm{b}} \simeq 10^{8} \mathrm{~m} / \mathrm{s}$ by the parameters of the two events (Battaglia \& Benz 2008). This indicates the beam electron density estimated by the average beam current $\left(\left\langle j_{\mathrm{b}}\right\rangle\right) n_{\mathrm{b}} / n_{0} \simeq 8.6 \times 10^{-3}$ for the event on Oct. 24,2003 and $n_{\mathrm{b}} / n_{0} \simeq 2.5 \times 10^{-3}$ for the event on Jul. 13, 2005, where $n_{0}$ is the background electron density in the loop plasma and reads as $\simeq 1.4 \times 10^{16} \mathrm{~m}^{-3}$ for the event on Oct. 24,2003 and $\simeq 3.2 \times 10^{16} \mathrm{~m}^{-3}$ for the event on Jul. 13, 2005 (Battaglia \& Benz 2008). To assume the density of the suprathermal electrons carrying the return current $n_{\mathrm{s}} \simeq 0.1 n_{0}$, the drift velocity of the return 
current (i.e., suprathermal) electrons $v_{\mathrm{d}}$ can be estimated by the current neutralizing condition $j_{\mathrm{r}}=j_{\mathrm{b}}$ as

$v_{\mathrm{d}}=\frac{n_{\mathrm{b}}}{n_{\mathrm{s}}} v_{\mathrm{b}} \simeq 8.6 \times 10^{6} \mathrm{~m} / \mathrm{s}$ and $2.5 \times 10^{6} \mathrm{~m} / \mathrm{s}$

for the events on Oct. 24, 2003 and on Jul. 13, 2005, respectively.

On the other hand, the saturation level $\alpha_{\mathrm{S}}$ for the ion-acoustic turbulence can be estimated by Eq. (9)

$\alpha_{\mathrm{S}} \equiv \frac{W_{\mathrm{ia}}^{\mathrm{S}}}{W_{\mathrm{ia}}^{\mathrm{T}}}=\frac{N_{\mathrm{D}}}{32 \pi} \frac{T_{\mathrm{e}}}{T_{\mathrm{i}}} \frac{v_{\mathrm{d}}}{v_{T_{\mathrm{e}}}} \sim 8 \times 10^{5}$ and $10^{5}$

for the events on Oct. 24, 2003 and on Jul. 13, 2005, respectively, which both are much greater than the necessary excited levels for the ion-acoustic turbulence, that is, $\alpha \sim 20$ and 7 for the events on Oct. 24, 2003 and on Jul. 13, 2005, respectively. The drift velocities $v_{\mathrm{d}}$ are much larger than the ion-acoustic velocity $c_{\mathrm{s}} \simeq 3.5 \times 10^{5} \mathrm{~m} / \mathrm{s}$, as well as that the saturation level of the ion-acoustic turbulence is much higher than the necessary excited level (i.e., $\alpha_{\mathrm{S}} \gg \alpha$ for both the events), indicates that the required exciting condition and strength for the ion-acoustic turbulence can easily be satisfied in these two events.

The return current $j_{\text {cr }}$ above is estimated on the basis of the classical ohmic law with the Coulomb collision $\left(v_{\mathrm{c}}\right)$. The latter is proportional to the Coulomb logarithm $\ln \Lambda$, which can, in general, have considerably different values at the fully ionized corona and at the partially ionized transition region and the chromosphere along the loop. The value of $\ln \Lambda \simeq 20$, which has been used in the above estimation, is usually valid for the fully ionized corona. In the partially ionized chromosphere, however, a lower value of $\ln \Lambda \sim 7$ is possibly more appropriate. This will result in a higher estimation of the return current $\left\langle j_{\mathrm{cr}}\right\rangle \sim 61.7$ and 20.6 for the events on Oct. 24, 2003 and on Jul. 13, 2005, respectively; as a result, a higher excited level of the ion-acoustic turbulence $\alpha \sim 61$ and 20 is required for the events on Oct. 24, 2003 and on Jul. 13, 2005, respectively. However, the required excitation level of the ion-acoustic turbulence is still much lower than the corresponding saturation level.

On the other hand, it is also possible that the plasma inhomogeneity from the fully ionized corona to the partially ionized transition region and the chromosphere along the loop significantly influences the excited ion-acoustic turbulence. For instance, the exciting condition of the ion-acoustic turbulence requires a current drift velocity of the return current that is much higher than the ion-acoustic velocity, which depends on the background density, as well as on the temperature in flares loops. The temperature drops, while the density increases steeply in the transition region down to the chromosphere. This is unfavorable for the excitation of the ion-acoustic turbulence because the increase in the density leads to a decrease in the current drift velocity. It is difficult to include changing density and temperature parameters in the present model. In the chromosphere, however, the electron-neutral collision can be expected to dominate the ohmic resistivity of the return current. Thus, the ion-acoustic turbulence possibly is not needed to produce a proper return current in the chromosphere.

\section{Summary}

Energetic electron beams are one of the most important products of solar flares. When propagating in the solar atmosphere and the interplanetary space they cause a series of effects by means of the interaction with the background plasma, such as the electromagnetic emissions in the radio and hard- $X$ bands, the excitation of plasma waves, and the induced electric field and return current. In turn, these effects can considerably influence the beam propagation, such as the energy loss and pitch-angle scattering of the beam electrons.

High-resolution observations of solar flares show that the spectral relations between the looptop and the footpoint sources can provide useful information about transporting processes of beam electrons and underlying microphysics in beam-plasma interaction in coronal loops. In this paper, on the basis of the two events presented by Battaglia \& Benz (2008), we estimated the drift velocity $\left(v_{\mathrm{d}}\right)$ of the electrons carrying the return current and found $v_{\mathrm{d}} \sim 8.6$ and $2.5 \times 10^{6} \mathrm{~m} / \mathrm{s}$ for the events on Oct. 24, 2003 and Jul. 13, 2005, respectively. They both are much higher than the ion-acoustic velocity $v_{\mathrm{s}} \sim 3.5 \times 10^{5} \mathrm{~m} / \mathrm{s}$ in these two events. This indicates that the current-driven instability can very effectively excite the ion-acoustic turbulence. To include the effects of the anomalous resistivity due to the ion-acoustic turbulence we further analyzed Ohm's law in the steady-state, beam-return current system. Our results showed that to satisfy the current neutralizing condition of $j_{\mathrm{r}}=j_{\mathrm{b}}$, the necessary excited level of the ion-acoustic turbulence $\alpha \equiv W_{\text {ia }} / W_{\text {ia }}^{\mathrm{T}} \sim 20$ and 7 for the two events mentioned above, respectively.

On the other hand, the saturation level of the corresponding ion-acoustic turbulence for these two events can be estimated by the weak-turbulence theory, and the result is $\alpha_{\mathrm{S}} \equiv W_{\mathrm{ia}}^{\mathrm{S}} / W_{\text {ia }}^{\mathrm{T}} \sim$ $8.6 \times 10^{5}$ and $10^{5}$ for above the two events, respectively. They both are much higher than the necessary excited level, implying that to explain the observed spectral evolution, the necessary excited condition and strength of the ion-acoustic turbulence both are easily satisfied in these two events. Thus, we show that including the anomalous resistivity by the ion-acoustic turbulence can successfully remove the contradiction found by Battaglia $\&$ Benz (2008), in which the current neutralizing condition is invalid because the estimated return current is much higher than the beam current. The results presented in this paper also indicate that the microscopic kinetics of plasma particles possibly play an important and critical role in understanding the dynamics of beam-return current systems in the solar atmosphere and the physics of solar flares.

Acknowledgements. The present research was supported by the NSFC under Grant Nos. 10973043 and 41074107, and by the MSTC under Grant No. 2011CB811402.

\section{References}

Arnoldy, R. L., Kane, S. R., \& Winckler, J. R. 1968, ApJ, 151, 711

Battaglia, M., \& Benz, A. O. 2006, A\&A, 456, 751

Battaglia, M., \& Benz, A. O. 2007, A\&A, 466, 713

Battaglia, M., \& Benz, A. O. 2008, A\&A, 487, 337

Benz, A. O. 2002, Plasma Astrophysics, Kinetic processes in Solar and Stellar Coronae, 2nd edn. (Dordrecht: Kluwer Academic Publishers)

Brown, J. C. 1971, Sol. Phys., 18, 489

Bret, A. 2009, ApJ, 699, 990

Cromwell, D., McQuillan, P., \& Brown, J. C. 1988, Sol. Phys., 115, 289

Emslie, A. G., Kontar, E. P., Krucker, S., \& Lin, R. P. 2003, ApJ, 595, L107

Feldman, U., Hiei, E., Phillips, K. J. H., Brown, C. M., \& Lang, J. 1994, ApJ, 421,843

Frost, K. J., \& Dennis, B. R. 1971, ApJ, 165, 655

Galeev, A. A., \& Sagdeev, R. Z. 1984, Current instabilities and anomalous resistivity of plasmas, in Basic Plasma Physics (New York: Elsevier), 271

Hannah, I. G., \& Kontar, E. P. 2011, A\&A, 529, A109

Hasegawa, A. 1975, Plasma Instabilities and Nonlinear Effects (Berlin, Heidlberg, New York: Springer-Verlag ), 217

Holt, S. S., \& Cline, T. L. 1968, ApJ, 154, 1027

Hoyng, P., Brown, J. C., \& van Beek, H. F. 1976, Sol. Phys., 48, 197 
Hoyng, P., Knight, J. W., \& Spicer, D. S. 1978, Sol. Phys., 58, 139

Hoyng, P., Duijveman, A., \& Machado, M. E. et al. 1981, ApJ, 246, L155

Huba, J. D. 2004, NRL Plasma Formulary (Washington, DC 20375: Naval Research Laboratory)

Hudson, H. S. 1978, ApJ, 224, 235

Karlicky, M. 2009, ApJ, 690, 189

Knight, J. W., \& Sturrock, P. A. 1977, ApJ, 218, 306

Labelle, J., \& Treumann, R. A. 1988, Space Sci. Rev., 47, 175

Larosa, T. N., \& Emslie, A. G. 1989, Sol. Phys., 120, 343

Lin, R. P., \& Hudson, H. S., 1976, Sol. Phys., 50, 153

Lin, R. P., Dennis, B. R., Hurford, C. J., et al. 2002, Sol. Phys., 210, 3

Masuda, S., Kosugi, T., Hara, H., Tsuneta,S., \& Ogawara, Y. 1994, Nature, 371 459

Masuda, S., Kosugi, T., Hara, H., et al. 1995, Publ. Astron. Soc. Jpn, 47, 677

Masuda, S., Sato, J., Kosugi, T., \& Sakao, T. 2000, Adv. Space Res., 26, 493

Papadopoulos, K. 1977, Rev. Geophys. Space Sci., 15, 113
Papadopoulos, K., \& Rowland, H. L. 1978, J. Geophys. Rev., 83, 5768 Petrosian, V., Donaghy, T. Q., \& McTierman, J. M. 2002, ApJ, 569, 459 Rowland, H. L., \& Vlahos, L. 1985, A\&A, 142, 219

Saint-Hilaire, P., Krucker, S., \& Lin, R. P. 2008, Sol. Phys., 250, 53

Sagdeev, R. Z. 1967, Am. Math. Soc. Providence, R. I., 281

Sagdeev, R. Z. 1969, Nonlinear Plasma Theroy (New York: Benjamin), 55

Spicer, D. S. 1977, Sol. Phys., 54, 379

Spicer, D. S., \& Sudan, R. N. 1984, ApJ, 280, 448

Su, Y., Holman, G. D., Dennis, B. R., Tolbert, A. k., \& Schwartz, R. A. 2009, ApJ, 705, 1584

Syrovatskii, S. I., \& Shmeleva, O. P. 1972, Soviet Astr. -AJ, 16, 273

Takakura, T. 1969, Sol. Phys., 6, 133

van den Oord, G. H. J. 1990, A\&A, 234, 496

Vlahos, L., \& Rowland, H. L. 1984, A\&A, 139, 263

Wheatland, M. S., \& Melrose, D. B. 1995, Sol. Phys., 158, 283

Zharkova, V. V., \& Siversky, T. V. 2011, ApJ, 733, 33 